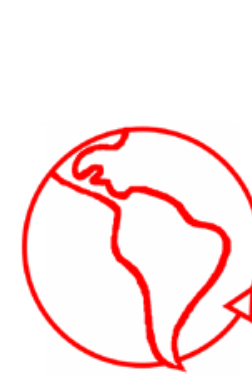

\title{
Acerca del concepto de sistema: Desde la observación de la totalidad hasta la totalidad de la observación
}

\author{
On the concept of system: from the observation of the totality to \\ the totality of the observation
}

\section{Guilherme Brandão}

Universidad Federal del Rio Grande del Sur, Brasil

\section{Resumen}

\begin{abstract}
El presente artículo analiza la emergencia del paradigma sistémico en el contexto de la evolución del concepto mismo de sistema. En ese sentido recorre la historia y evolución del concepto de sistema, los diferentes conceptos y teorías científicas asociadas a este concepto. Se analizan las raíces filosóficas del concepto, los diferentes momentos de la teoría de sistemas, la teoría general de sistemas, las diferentes corrientes cibernéticas y finalmente la teoría de Niklas Luhmann. El artículo concluye que la teoría de sistemas de Luhmann constituye una progresión y a la vez un cambio paradigmático en la teoría de sistemas.
\end{abstract}

\section{Abstract}

This article analyzes the emergence of systemic paradigm in context of the evolution of the concept of system. In this sense traces the history and evolution of the system concept, the different concepts and scientific theories associated with this idea. It analyzes the philosophical roots of the system concept, the different moments of the theory of systems, the general systems theory, the different currents of cybernetic thought and finally the theory of Niklas Luhmann. The article concludes that the systems theory of Luhmann is a progression and also a paradigmatic change in system theory.

\section{Palabras Clave: Sistema; Totalidad; Cibernética; Cibernética de Segundo Orden; Niklas Luhmann}

Keywords: System; Totality; Cybernetics; Second Order Cybernetics; Niklas Luhmann

\section{Introducción}

Desde un punto de vista algo simplificado, se puede señalar que la ciencia posee tres características fundamentales, a saber: la universalidad de su objeto, el empleo de un método y sistematicidad en la producción de conocimiento. Es decir, mediante el uso de un método particular, son producidas leyes científicas universales, desde las cuales se pueden producir teorías, tesis o hipótesis. El producto de la utilización de tal método producirá una totalidad orgánica, y sólo a partir de ese punto, existirá ciencia.

"Los conocimientos constituyen ciencia en el momento en que, más allá de que son universales, se articulan orgánicamente en un conjunto coherente, en el cual demuestran la interdependencia y la conexión necesaria que tienen unos en relación con los otros." (Enciclopedia Mirador Internacional 1981: 10467) 
Dicha articulación orgánica es lo que comúnmente se conoce como sistema. El término sistema tiene su origen en el griego synhistanai ("poner junto"). En sentido amplio significa "un conjunto o una totalidad de objetos, reales o ideales, recíprocamente articulados e interdependientes, uno en relación a los otros." (Enciclopedia Mirador Internacional 1981: 10465).

A pesar de que la sistematicidad está relacionada con el núcleo del concepto de ciencia desde Aristóteles (la ciencia debe basarse en la organicidad de su "corpus", en interconexión con el conocimiento obtenido por el uso de sus métodos) demoró siglos en desarrollarse la utilización de métodos científicos con el fin de extraer el conocimiento contextual, holístico del objeto de estudio, haciendo hincapié en la extracción de conclusiones científicas, no mediante el estudio de sus partes de manera aislada, sino a partir del estudio de su totalidad. La adopción de la sistematicidad -inmanente al concepto de ciencia- para su práctica efectiva demoró el tiempo que se extiende desde de la Grecia antigua hasta nuestros días. Esta dificultad general de contextualización afecta más directamente (en principio) a las ciencias sociales que a las ciencias naturales. Esta adopción de la sistematicidad empieza a tomar fuerza en las ciencias sociales después de Hegel $y$, se fortalece en la segunda mitad del siglo XX, al alero de innumerables trabajos en diversos senderos de la ciencia.

\section{Del concepto de totalidad/sistema hasta el siglo XX}

En los tiempos de la filosofía presocrática -también llamada filosofía cosmológica ya que tiene como interés principal la naturaleza- los filósofos definían usualmente la totalidad mediante contradicciones. Un exponente ejemplar en ese contexto fue Heráclito de Éfeso, padre de la dialéctica, corriente filosófica que se basa en la idea de contradicción y oposición. La contradicción y la lucha eran para Heráclito la esencia de la vida. Heráclito planteaba que la totalidad o sistema era un producto de la realidad y del logos, y que el sistema estaba dotado de estructura contradictoria y cambiante. Entendía la naturaleza como un proceso "del absoluto como devenir y de la unidad o de la unificación de los opuestos". (Enciclopedia Mirador Internacional 1981: 3305).

Las ideas cosmológicas de la filosofía influyeron en un principio a Platón y Aristóteles, pero pronto perdieron su fuerza, a causa de la adopción del "principio de no contradicción" ${ }^{\prime \prime}$, el cual pasó a ser muy relevante en el pensamiento de la filosofía griega clásica y será a posteriori uno de los pilares fundamentales del concepto de ciencia (hasta la mecánica clásica newtoniana).

Para Aristóteles la totalidad/sistema encontraba su forma concreta en el concepto de naturaleza. Con esto, al concepto de sistema le fue añadido el concepto de orden eterno y racional, el cual estaba relacionado con la naturaleza en la concepción griega. El concepto de naturaleza emerge a partir de este concepto de orden. La naturaleza es en este sentido, una totalidad ordenada, donde no existe espacio para la contradicción. Lo que existe en la naturaleza está además racionalmente organizado.

Este concepto aristotélico de naturaleza como un todo ordenado o sistema marcará el paradigma de la ciencia moderna, el cual sumado al pensamiento cartesiano, tendrá influencia hasta las ideas de Newton. Esta concepción del mundo simbolizada por un orden estricto, por la rigurosidad, por la mecánica, es criticada por Morin: "La ley eterna que regula la caída de las manzanas ha suplantado la ley del Eterno que, por una manzana, hizo caer a Adán." (Morin 2001: 50)

\footnotetext{
${ }^{1}$ El "principio de no contradicción" es un antiguo principio lógico que sostiene que una proposición cualquiera y su respectiva negación, no pueden ser ambas verdaderas en el mismo sentido y de manera simultánea. (ver Purtil 1999: 737)
} 
En el periodo de la emergencia de la mecánica newtoniana, la totalidad ya no se refiere más al organismo, sino a la máquina cognoscible por la razón. En ese contexto emerge el término mecanismo. Así, lo plantean Arshinov y Fuchs:

"La visión del mundo newtoniana estaba caracterizada por su creencia en la absoluta inmutabilidad de la naturaleza y por una metodología reduccionista. La naturaleza era considerada como un sistema conservador que permanece estable desde sus inicios hasta su final, el tema orgánico se redujo a la mecánica." (Arshinov \& Fuchs 2000: 200)

A pesar de la aparente tranquilidad alcanzada por el pensamiento cartesiano, aparece una nueva voz que empieza a exponer rasgos de lo que podría ser la ciencia moderna. Esta nueva voz será Blaise Pascal.

Pascal expone un mundo nuevo, una nueva realidad basada en aquella paradoja insuperable que es el hombre. En ese contexto, emergen conceptos novedosos y complejos vigentes hasta hoy en día. Pascal dialogaba con la ciencia a través de la firme convicción de la existencia de un universo infinito, un universo más allá vislumbrado no en partes sino en su entereza, de manera sistémica y contextual, al alero de una racionalidad difícil de ser observada incluso por el pensamiento actual: "no procuremos por lo tanto, seguridad y firmeza, nuestra razón está siempre decepcionada por la inconstancia de las apariencias: nada puede fijar el finito entre los dos infinitos que lo encarcelan y huyen de él." (Pascal 2001: 83)

Pascal ubicaba la dialéctica de Heráclito en un nivel diferente, en un camino alrededor de algo que nos remite al isomorfismo, a una similitud tanto epistémica respecto de la urdidumbre propia de las cosas: "La inmovilidad fija y constante de la naturaleza, en comparación con el cambio continuo que sucede en nosotros, debe causar los mismos efectos." (Pascal 2001: 84)

Pascal define por primera vez la complejidad. Dicha definición se ubica en clave sistémica y contextualizada en un ambiente difícil de imaginar antes de la modernidad: "El mundo visible (...) es una esfera infinita, cuyo interior está por todas partes, y la circunferencia en ninguna." (Pascal 2001: 79)

Pascal hace hincapié de esta manera en la totalidad como un conjunto interdependiente que se compone de un todo mayor, en partes que se mezclan y se extienden en un mundo novedoso. En ese contexto se puede observar que la capacidad de la ciencia para proponer leyes generales y conocer la totalidad de las cosas bajo la observación de partes aisladas, no parece tener más sentido. La ciencia empieza a re-entrar ${ }^{2}$ la organicidad -es decir la sistematicidad- en el seno de la ciencia, como un concepto todavía poco delineado de sistema. Dicho de otra manera, el concepto de sistema empieza a generar posibilidades epistemológicas interesantes para ser observado recursivamente en la historia de la ciencia.

Estos lineamientos de la interdependencia, como parte constituyente del concepto de totalidad y sistema -ya delineados por Heráclito y Pascal- serán desarrollados por Hegel y los materialistas que vienen después de él.

Prigogine y Stengers (1984) señalan de manera concisa la importancia de los filósofos alemanes que abrazaron la dialéctica. Es decir, los conceptos de auto-organización y emergencia empiezan a ser dibujados desde allí.

\footnotetext{
${ }^{2}$ En el sentido de Re-Entry de Spencer-Brown (1979)
} 
"La filosofía hegeliana de la naturaleza incorpora sistemáticamente todo lo que es negado por la ciencia newtoniana. En particular, se basa en la diferencia cualitativa entre el comportamiento simple, descripto por la mecánica y el comportamiento de entidades más complejas, como los seres vivos. Ella niega la posibilidad de reducir esos niveles, rechazando la idea de que las diferencias son solo aparentes y que la naturaleza sería básicamente homogénea y sencilla." (Prigogine \& Stengers 1984: 89)

"La idea de historia de la naturaleza como parte integral del materialismo fue afirmada por Marx $y$, en mayor detalle, por Engels. Los desarrollos contemporáneos de la física, el descubrimiento del rol constructivo desempeñado por la irreversibilidad, han planteado dentro de las ciencias naturales una pregunta que siempre se hecho entre los materialistas. Para ellos, la comprensión correcta la naturaleza significaba comprenderla como capaz de producir al hombre y a la sociedad." (Prigogine \& Stengers 1984: 252)

Hegel, el representante más completo del idealismo alemán y el primer filósofo de la dialéctica moderna ha contribuido en gran medida para la construcción contemporánea del concepto de sistema. Con su trabajo, crítico del mecanicismo y dialéctico por principio, pretendió -y logró- exponer un concepto de sistema que buscaba aprender el todo en su totalidad: "en ningún lugar, ni en el cielo ni en la tierra, hay algo que no contenga en sí a ambos, el ser y la nada" (Hegel 1982: 41). Conforme se lee en la editorial de la prestigiosa revista Anthropos (para los números 173 y 174 de 1997): "fue Hegel quien se atrevió a proponer simultáneamente una teoría de la ciencia, de la sociedad, de la conciencia y del mundo". (Anthropos 1997: 8)

Federich Engels tuvo gran contribución en la formalización de un concepto de ciencia que observa el objeto de estudio en todos sus matices, en su totalidad, en la interconexión de sus partes más pequeñas formando una totalidad compleja en cambio continuo. La concepción de naturaleza en Engels se refiere a este punto:

"Toda la naturaleza asequible a nosotros forma un sistema, una concatenación general de cuerpos, entendiendo aquí por cuerpos todas las existencias materiales, desde los astros hasta los átomos, más aún hasta las partículas del éter, de cuanto existe. El hecho de que estos cuerpos aparezcan concatenados lleva ya implícito el que actúan los unos sobre los otros, y en esta su acción mutua consiste precisamente el movimiento." (Engels 2000: 42)

Engels, admitiendo un conjunto, donde las partes actúan unas sobre las otras, expone la interdependencia inherente a las partes de un sistema conforme la concepción contemporánea. Este autor, muy por delante de su tiempo, fue uno de los precursores de la idea de complejidad en el siglo XIX y profundiza de cierta manera el hegelianismo, anticipándose a las teorías que trascienden las fronteras disciplinarias. Kinouchi reitera este hecho:

"Engels estaba fascinado por las propiedades emergentes y transiciones de fase reveladas por la termodinámica y por la física estadística y trata de aplicar estos conceptos (no sólo metafóricamente) a la economía, la historia y otras ciencias sociales. Hace hincapié en que la evolución de la materia se da históricamente por transiciones entre niveles cualitativamente diferentes de organización. Su punto de vista de los procesos naturales y sociales es dinámico, sistémico y ecológico: Engels define la dialéctica como la ciencia general de los cambios y de las interconexiones." (Kinouchi 2006: 4)

En este contexto ingresa el pensamiento sistémico el siglo XX con posibilidad de absorber, mediante el concepto de sistema, la negación y -si se piensa a partir de Pascal- caminar 
hacia un orden no-jerárquico, donde no hay sitio para un centro o un control. Junto con esto la ciencia clásica pierde su fuerza epistemológica. Todo este potencial de cambio será utilizado de buena manera a partir de la emergencia de la cibernética.

\section{A partir del siglo XX}

La necesidad de exponer el objeto de estudio en su contexto y proporcionar herramientas sistémicas más allá de un contexto disciplinario, da lugar al nacimiento de la llamada Teoría General de Sistemas (TGS) ideada por Ludwig von Bertalanffy y también por Ralph Gerard, Keneth Boulding y Anatol Rapopport.

Bertalanffy, como afirma Esteves, era más que un biólogo, "él era un científico en un sentido mucho más amplio, preocupado con cuestiones que trascienden las fronteras disciplinarias" (Esteves 2005: 195). El autor austriaco, concibió, basándose en el concepto de sistema, un mecanismo de integración entre las ciencias naturales y sociales. Dicho mecanismo funcionaría sobre las bases de las uniformidades estructurales que se pueden observar en los diferentes senderos de la ciencia, buscando su unificación a través de conceptos y métodos unificadores los que, sin embargo, no eliminaban las diferencias esenciales.

Como exponen Rodríguez y Arnold: "La obra de Bertalanffy tiene un enorme impacto en la investigación y búsqueda de un marco de referencia que permita el estudio y comunicación de los diversos ámbitos y temas científicos" (Rodríguez \& Arnold 2007: 41). A pesar de este gran paso, la perspectiva epistemológica de la TGS fue construida en gran medida al alero del paradigma mecanicista pues Bertallanfy buscaba estructurar su teoría a la similitud de la física newtoniana, modelo mayor del mecanicismo.

En ese contexto, emerge la concepción de sistema abierto, pensado bajo el modelo de causa y efecto, es decir el sistema se orienta teleológicamente a adaptarse al entorno donde está inserto. Esta estructura en sí no tendría, a final de cuentas, diferencias mayores respecto de las concepciones sistémicas del clasicismo y, por otro lado, en comparación con las ideas de Pascal, Hegel o Engels no se observa una gran innovación. Sin embargo, en base a la idea de isomorfismo característico de esa teoría hubo avances que se verifican con vigor cuando se observan los arreglos colaborativos de multi- inter- y transdisciplinariedad en sus investigaciones- aunque sus concepciones epistemológicas no respondían a los desafíos de su época. Si se analiza el concepto de sistema abierto al alero de la crisis de representación de la ciencia, vale decir, a partir de la emergencia de la complejidad, la TGS parece tener poca potencia, pues esta idea de sistema está basada en una linealidad y como modelo analítico, por ejemplo, a partir del concepto de evolución, no parece dar nuevos avances. Estas falencias son señaladas de manera mucho más clara a posteriori, especialmente a partir de los trabajos de la cibernética de Norbert Wiener y otros científicos.

Pocos años después del surgimiento de la TGS, emergen los esfuerzos conjuntos de un conjunto de científicos de diversas disciplinas, quienes desarrollan lo que sería denominado cibernética ${ }^{3}$. La cibernética, según la definición de Norbert Wiener, es la

\footnotetext{
${ }^{3}$ Este movimiento intelectual se desarrolló con el apoyo de las Conferencias Macy que tuvieron lugar en Nueva York desde 1946. Organizadas por Frank Fremont-Smith, concurrieron a estas conferencias entre otros, Norbert Wiener y Von Neumann en relación con las matemáticas, y Gregory Bateson y Margaret Mead de las ciencias sociales. Vale la pena destacar la prominencia de Norbert Wiener, quien se erigió como una figura dominante en toda la serie de diez conferencias que se celebraron. Gracias al diálogo dado en estos encuentros se produjeron interesantes avances para la ciencia, entre ellos podemos mencionar: la construcción de la primera computadora, el desarrollo y discusión de la teoría matemática de la comunicación de Shannon y Weaver, las ideas iniciales que luego dieron pie al descubrimiento del ADN, las ideas de la biología de la cognición que posteriormente desarrolló Humberto Maturana, conceptos relativos
} 
ciencia del control y de la comunicación en el animal y en la máquina. Ashby, otro gran exponente de la cibernética plantea, por su parte, que la Cibernética es: “...también, una teoría de las máquinas, pero no se ocupa de las cosas, sino formas de comportamiento de ellas. No pregunta "¿qué es esta cosa?" sino "¿qué hace?" (Citado en Umpleby 2000). Así, está más interesada en una proposición como "esta variable se somete a una oscilación armónica simple", y se preocupa menos por si la variable se ubica en una posición en un punto sobre una rueda o si se trata de un potencial en un circuito eléctrico. Por tanto, es esencialmente funcional y comportamental." (Citado en Umpleby 2000)

Maturana ha propuesto la siguiente definición para el término cibernética:

“He propuesto para la cibernética la frase: "el arte y la ciencia de la comprensión humana" ¿Por qué? Porque la persona que dirige la nave, el capitán, actúa tanto sobre un know- how práctico como por intuición. Por lo tanto, el comandante actúa como un científico y un artista (...) Comprender un sistema requiere tanto intuición como comprensión gestáltica de las coherencias sistemáticas del sistema considerado, y mirar las coherencias estructurales (causales) del lugar donde está ubicado el observador. Comprender involucra, además, relacionar estas dos perspectivas operacionales diferentes de manera que, aunque no deductivamente, muestre la conectividad dinámica de cualquier parte del sistema hasta la dinámica total del sistema. De este modo, en la medida en que la cibernética tiene que ver con el tratamiento de sistemas, así como con su explicación científica a medida que vayan surgiendo para nuestro entendimiento como observadores, es que yo denomino a la cibernética el arte y la ciencia de la comprensión." (Citado en Umpleby 2000)

Debido a la amplitud de definiciones que se conocen, en el presente artículo operacionalizaremos la postura de Gordon Pask para la comprensión del concepto de sistema en la cibernética:

"El ciberneticista tiene su campo de interés bien definido, a pesar de que sea gigante. El objeto de su estudio es todo el sistema artificialmente construido o resultante de la abstracción de la estructura física de un sistema natural, el cual exponga la interacción entre sus partes, de manera que unas controlen las otras, independientemente del carácter físico de esas partes. Él trabaja y cambia sus sistemas utilizando muchas de las veces, técnicas matemáticas. Como en casos prácticos la cibernética es muy útilmente aplicada a sistemas enormes, el podrá también construir dispositivos físicos para modelarlos. (Pask 1970: 35- 36)

Al alero de todo lo anteriormente mencionado, es posible apreciar el esfuerzo conjunto de un gran número de científicos involucrados en la cibernética. Desde las conferencias Macy ${ }^{4}$ hasta la llamada cibernética de segundo orden el concepto de sistema cambia de manera radical. Estos cambios epistemológicos fueron interesantes en cantidad y de calidad notable, algo que merece, ciertamente, aún más investigaciones.

Esta contextualización sirve para ilustrar de mejor manera la evolución del concepto de sistema, en el periodo ubicado entre la década de los 40's hasta nuestros días.

\footnotetext{
a la inteligencia artificial desarrollados por Herbert Simon y los escritos acerca del concepto de decisión que le valieron a este último un premio Nobel.

${ }^{4}$ La cibernética existe desde antes de las conferencias Macys. Un ejemplo usualmente citado es la publicación de "Behavior, Purpose, and Teleology" de Norbert Wiener, Julian Bigelow y Arturo Rosenblueth en 1943.
} 


\section{El sistema en la cibernética y cibernética de segundo orden}

A la luz de las definiciones anteriores, resulta evidente que sea cual fuere la definición de cibernética, su operacionalización ocurre al alero del concepto de sistema. Por este motivo, este concepto cambia a lo largo del desarrollo de la disciplina. De manera recíproca: lo que ocurre en la cibernética ocurre en el sistema. Por otro lado, debido a su carácter transdisciplinario el concepto de isomorfismo adquiere una importancia central. De manera también recíproca: la operacionalización (sistema) opera bajo el isomorfismo. Así, por así decir, en el ADN de la transdisciplina se ubica la relación interdependiente entre sistema e isomorfismo. Dicha relación es uno de los grandes aportes de la TGS, además de otros conceptos como sinergia y equifinalidad.

En la cibernética la causalidad adquiere importancia, aunque de manera no- lineal. El trabajo de los ciberneticistas está relacionado con la circularidad y especialmente con la información acerca de dicha circularidad. De este modo, se buscaba desmenuzar el concepto de teleología, hasta que una vez este concepto relativamente desconfigurado abriera espacio para producir información acerca de todos los estados posibles de un sistema. Como nos enseñan Rodríguez y Arnold: "Se trata en definitiva, de una nueva teleología, donde las formas de organización y las metas (outputs) se definen en su relación mutua" (Arnold \& Rodriguez 2007: 42).

En el contexto anterior, es decir de causalidades circulares, no es difícil imaginar que la cibernética operaría casi en concordancia con la idea de un sistema abierto (como en Bertalanffy). No obstante lo anterior, puede surgir la pregunta acerca de si las entradas de información (inputs) se relacionan con las salidas (outputs) y, de suceder esto: ¿qué ocurre en el sistema? O dicho de otro modo: ¿en qué medida ocurre una relación entre sistema y entorno? Estas y otras interrogantes conducen al surgimiento de cibernética de segundo orden.

Sin embargo, huelga señalar que en este punto se abandona en gran medida la perspectiva newtoniana. Para el postulado lógico de la no-contradicción, el efecto o es efecto o es nada. Pero en el contexto de la cibernética, poco importa si es efecto o si es causa de algo, lo que importa es la información que se obtiene con el feedback. La contradicción está por lo tanto en el seno de una ciencia probabilística. La verdad absoluta, otro pilar del clasicismo, no está más en juego. Así, resulta fundamental comprender que el concepto de sistema generaliza la idea de información, sin embargo aplicada para la búsqueda de información probabilística, no de una verdad absoluta del mundo. Se sabe que no se puede saber la totalidad de las cosas.

Lo que opera, se operacionaliza en el seno de un concepto de sistema, los estados observados son emergencias, vale decir, son producto de la interacción entre partes que operan en conjunto y que son interdependientes. La posibilidad de análisis, de observar en partes aisladas -en un sentido cartesiano- es la excepción. La regla ahora es la información contextual de un sistema que opera. Sin embargo opera en respuesta a un ambiente. ¿Hasta cuándo?

En la llamada segunda cibernética, la operacionalidad del sistema empieza a ser observada a partir del feedback positivo, que tiene relación de manera más potente con las interconexiones entre las partes constituyentes de un sistema. Lo que se produce son posibilidades de generación de información acerca de: "la generación de nuevas formas, la diferenciación, el crecimiento, la acumulación" (Maruyaman 1968: 304. Citado en Rodríguez \& Arnold 2007: 45), es decir, la apertura del sistema empieza a cerrarse pero nunca de manera completa. El foco de observación se dirige ahora hacia la interioridad del sistema. 
Lo importante en ese sentido no es, sin embargo, ni el cierre ni la apertura. Lo que se puede observar es la pérdida de importancia de cualquier concepto de teleología -la cual ya había perdido ciertamente fuerza en la primera corriente de la cibernética- y además la decreciente importancia del concepto de entorno y su capacidad de control, un concepto que era central para la primera cibernética. En esta nueva corriente el entorno es visto como un factor menos determinante de la operacionalidad del sistema. Pero ¿quién produce la producción? Ahora la -por así decir- poliarquía de Pascal empieza a tener mucho sentido práctico, pues no es que no exista un centro de control, el centro está en muchos sitios, la contingencia es un hecho.

En ese punto de la historia de la cibernética, las figuras más destacadas son las de Heinz von Foerster y los biólogos chilenos Humberto Maturana y Francisco Varela. La cibernética es ahora una cibernética de la cibernética, la forma cibernética re-ingresa en la forma cibernética. El sistema re- ingresa en el sistema, y en ese sentido si se piensa en Hegel padre fundador de esta idea- nos encontramos en la búsqueda del espíritu, es decir de la racionalidad. ¿Cómo opera cuando opera?, ¿bajo qué "racionalidad" hace lo que hace? En ese sentido, cuando la operación sistémica operacionalizada en el sistema como modelo se extiende hasta su punto máximo, la sociología, una de las dueñas de la complejidad organizada (a decir de Weaver), empieza, en alguna medida, a mirar de manera más desmenuzada el concepto de sistema.

En ese contexto Walter Buckley, bajo el concepto de sistema adaptativo complejo, plantea lo siguiente:

\begin{abstract}
"Sólo los sistemas cerrados que llegan hasta los estados más probables, es decir, la pérdida de la organización y la energía disponible, pueden ser tratados de manera rentable en términos de equilibrio. Fuera de este contexto, el concepto de equilibrio parece bastante inapropiado y sólo es útil engañosamente. Por otro lado, sólo los sistemas abiertos, adaptativos complejos, que pueden ser tensionados y son flexibles pueden elaborar y proliferar la organización. Cannon acuñó el término "homeostasis" para los sistemas biológicos de modo de evitar las connotaciones de equilibrio y para mostrar las propiedades de mantenimiento potencial dinámicas y procesuales de sistemas fisiológicos básicamente inestables. Al tratar con el sistema socio-cultural, sin embargo, necesitamos de un nuevo concepto para expresar no sólo la función de mantenimiento estructural, sino también la estructura de elaboración y el cambio de función de sistemas inherentemente inestables." (Buckley 1968: 493)
\end{abstract}

Las concepciones de von Foerster, Maturana y Varela, al alero del Ilamado también constructivismo radical caminan por ese sendero, donde la causalidad es reemplazada por la objetividad y la trivialización, donde los sistemas son auto-referentes y observan sistemas que observan sistemas.

Niklas Luhmann finaliza epistemológica- y sociológicamente este modelo en Sistemas Sociales (1998), señalando a la sociedad como un sistema auto-referente, que se autoproduce al alero de la comunicación, la cual a su vez se reproduce de manera autopoiética, y dejando al antiguo concepto sociológico de acción con la importante función de operacionalizar la auto- observación y la auto- descripción de los sistemas.

\title{
Conclusión: El concepto de sistema en Luhmann y un cambio paradigmático
}

El sistema en Luhmann es una diferencia producida por el sentido presente en la comunicación. La totalidad deviene sistema, de modo que la concepción de fronteras, de límites -como cierre para producir la identidad que se llama sistema- adquiere importancia para la construcción del concepto. Al igual que para Pascal, el centro, la 
concepción autárquica, no tiene importancia. Al igual que para Engels, la contradicción, la inclusión de la negación, es fundamental para el modelo de sistema.

Luhmann recoge de Bertalanffy la sistematicidad de una nueva ciencia emergente, es decir, la mayor operacionalidad entre fronteras disciplinares bajo conceptos comunes y el entorno vuelve a tener una importancia fundamental (señalada primeramente por la primera cibernética).

Sin embargo, para Luhmann es crucial la observación de esa totalidad en otro nivel cualitativo de emergencia. El cierre del sistema, que no es completamente un cierre, permite la entrada de algo que potencialmente informa, que diferencia -en la medida que se observa un sentido allí- y produce lo que lo produce, a partir de la diferencia entre una unidad de una diferencia (sistema) y su entorno. Es difícil en ese sentido no apreciar que, de manera algo paradojal, el sistema es una desconstrucción emergente que construye algo en sus operaciones temporales.

Como conclusión, resulta fundamental comprender que la información que se produce en la autoproducción de un sistema es información para y a partir de un observador. El sistema como diferencia, es producto de la observación de observaciones que producen y auto- producen cristalizaciones de sentido. En este gran cambio epistemológico no existe ontología ni teleología, lo que existe como modelo que genera información probabilística y contingente, es la operación incluida o excluida por la autopoiesis de un sistema, siempre como diferencia apuntada por un observador. Un sistema es la unidad de la diferencia sistema -él mismo- y entorno a partir de una distinción, a partir del ojo del observador.

En ese sentido, se pueden observar las diferencias entre grados de complejidad en la operacionalidad de un sistema. Sistema es para Luhmann primeramente diferencia. Es operacionalización de probabilidades en un escenario donde la reducción de complejidad no significa reduccionismo, puesto que siempre es contingente y abierta. Sin embargo hay un hilo observable recursivamente que informa y hace que el concepto de sistema, siempre en un sentido cognitivo, genere condiciones de posibilidades para la producción de episteme al alero de cristalizaciones de sentido (semántica). Conocimiento científico bajo otros principios epistemológicos. RM

\section{Bibliografía}

Anthropos (1997). Editorial. La sociedad como Teoría de Sistemas autorreferenciales y autopoieticos de comunicación. Nuevos presupuestos críticos, nuevos conceptos e hipótesis en la investigación sociológica en la sociedad contemporánea. Anthropos $173 / 174$.

Arshinov, V. \& Fuchs, C. (2003). Causality, Emergence and Self-Organization http:/ / www.self- organization.org/ results/ book/ EmergenceCausalitySelfOrganisation.pdf (Consultado el 1 de Marzo de 2008)

Buckley, W. (1968). Society as a Complex Adaptive System. En W. Buckley (Ed.), Modern Systems Research for the Behavioral Scientist (pp. 490- 513). Chicago: Aldine.

Enciclopedia Mirador Internacional (1981). São Paulo: Enciclopaedia Britannica do Brasil.

Engels, F. (2000). A Dialética da Natureza. São Paulo: Editora Paz e Terra.

Esteves, M. J. (2003). Pensamento Sistêmico: o novo paradigma da ciência. Campinas: Editora Papirus. 
Hegel, G.W.F. (1982). Ciencia de la Lógica. Bogotá. Ediciones Solar.

Kinouchi, O. (2008). Um Precursor das Ciências da Complexidade no Século XIX http://arxiv.org/pdf/physics/0110041.pdf (Consultado el 2 de Mayo de 2009)

Luhmann, N. (1998). Sistemas Sociales: Lineamientos para una teoría general. Barcelona: Anthropos.

Morin, E. (2001). El método I. La naturaleza de la naturaleza. Madrid: Cátedra.

Pascal, B. (2001). Pensamentos. São Paulo: Ed. Martins Fontes.

Pask, G. (1970). Uma introdução à Cibernética. Coimbra. Arménio Amado Editor.

Prigogine, I. \& Stengers, I. (1984). Order out of Chaos. New York. Bantam.

Purtill, R. (1999). Principle of Contradiction. En R. Audi (Ed.), The Cambridge Dictionary of Philosophy (p. 737). New York: Cambridge University Press.

Rodríguez, D. \& Arnold, M. (2007). Sociedad y teoría de sistemas. Elementos para la comprensión de la teoría de Niklas Luhmann. Santiago de Chile: Editorial Universitaria.

Spencer- Brown, G. (1979). Laws of Form. New York: E.P. Dutton.

Umpleby, S. (2000). Defining 'Cybernetics'.

http:// www.asc- cybernetics.org/foundations/ definitions.htm (Consultado el 5 de Abril de 2009)

\section{Sobre el autor}

Guilherme Brandão Licenciado en Derecho en la Universidad UNISANTOS (Brasil) y Magíster en Análisis Sistémico Aplicado a la Sociedad de la Universidad de Chile. Actualmente cursa el doctorado en sociología en la Universidad Federal del Rio Grande del Sur (Brasil) como becario CAPES.

guisnbbrandao@gmail.com

Contacto

Instituto de Filosofia e Ciências Humanas - Programa de Pós- Graduação em Sociologia Doutorado

Conceito CAPES (2007- 2009): 6

Endereço: Av. Bento Gonçalves, 9500

Porto Alegre - Rio Grande do Sul

Brasil

Recibido: Febrero 2012

Aceptado: Abril 2012 